\title{
Comparación de dos procedimientos de enseñanza universitaria: Un ejemplo de interteaching
}

\author{
Yors A. Garcia \\ Fundacion Universitaria Konrad Lorenz - Bogotá - Colombia \\ Laurent Orozco \\ Fundacion Universitaria Konrad Lorenz - Bogotá - Colombia \\ Giouvanni Martin \\ Fundacion Universitaria Konrad Lorenz - Bogotá - Colombia
}

\section{Resumen}

El objetivo de ésta investigación fue comparar dos procedimientos de enseñanza universitaria en el rendimiento académico. Un total de 11 estudiantes fueron evaluados en las dos condiciones experimentales, interteaching con discusión por pares (ICDPP), e interteaching sin discusión por pares (ISDPP). Se utilizó un diseño de tratamientos alternantes para comparar ambas condiciones. En cada condición los participantes completaban unas guías de estudio, en la condición de (ICDPP) los estudiantes discutían en parejas los temas de las guías y seleccionaban los de la siguiente clase. Posteriormente el docente revisaba los temas de la clase anterior y administraba un quiz al final. La condición de ISDPP fue idéntica a la anterior, con la excepción que no se presentaba la discusión por pares. Los resultados muestran que las notas más altas en los quices ocurrieron en la condición de ICDPP. Se discuten los resultados y se presentan alternativas de investigación.

Palabras Clave: Métodos de enseñanza; análisis de la conducta; educción superior.

\section{Comparison of two procedures of college teaching: An example of interteaching}

\begin{abstract}
The objective of this study was to compare two methods of college teaching in increasing quizzes scores. A total of 11 students were tested in the two experimental conditions, interteaching with peer discussion (IWPD) and interteaching without peer discussion (IWOPD). An alternating treatment design was used to compare both procedures. In each condition participants completed study guides before class, then in the IWPD students meet in groups to discuss and selectt eaching topics for class. This condition ended with a quiz. The other condition, IWOPD was similar to the above, except students did not meet in groups to discuss the study guides. The results show that quiz scores were higher in the ICWPD. Some educational implications are presented and futures research described.
\end{abstract}

Keywords: Teaching methods; behavior analysis; higher education.

\section{Comparação de dois procedimentos de ensino universitário:}

\section{Um exemplo de interteaching}

\section{Resumo}

O objetivo desta investigação foi comparar dois procedimentos de ensino universitário relativos ao rendimento acadêmico. 11 estudantes foram avaliados em duas condições experimentais: interteaching com discussão por pares (ICDPP) e interteaching sem discussão por pares (ISDPP). Foi utilizado um delineamento de tratamentos alternados para comparar ambas as condições. Em cada condição os participantes completavam guias de estudo; na condição de ICDPP os estudantes discutiam em pares os temas das guias e selecionavam os da aula seguinte. Posteriormente o docente revisava os temas da aula anterior e administrava um questionário ao final. A condição de ISDPP foi idêntica à anterior, com a exceção que não se apresentava a discussão por pares. Os resultados mostram que as notas mais altas nos questionários ocorreram na condição de ICDPP. Discutem-se os resultados e se apresentam alternativas de investigação.

Palavras-chave: Métodos de ensino; análise da conduta; ensino superior. 


\section{Introducción}

La psicología comportamental ha realizado grandes avances en las últimas décadas, entre ellas está la aplicación de la tecnología operante a la educación, y en particular a la enseñanza universitaria (Bernstein \& Chase, 2013; Henklain \& Carmo, 2013). Desde sus inicios el análisis de la conducta, que es una rama de la psicología comportamental, la cual cuenta con su propia epistemología, metodología y procedimientos de intervención, ha desarrollado varias tecnologías de enseñanza aplicadas a la educación superior. En los años 50s se realizaron las primeras aplicaciones de la tecnología operante al campo de la educación con el diseño y uso de las máquinas de enseñanza en la educación básica (Ferster, 2014), estas posteriormente evolucionaron hasta convertirse en elementos centrales en el proceso de formación académica (Kara \& Sevim, 2013; Rehfeldt, Jung, Aguirre, Nichols, \& Root, 2016). El eje fundamental de las máquinas de enseñanza ha sido la instrucción programada, la cual hace uso de varios procedimientos operantes (reforzamiento, moldeamiento, desvanecimiento, etc.) para el entrenamiento de habilidades académicas en diferentes poblaciones y contextos.

Durante los mismos años de desarrollo de la instrucción programada aparecieron métodos de enseñanza académica basados en la tecnología operante, como la enseñanza en precisión de respuesta (precisión teaching) (Lindsley, 1991), y el sistema de instrucción personalizada (personalized system of instruction) (Keller, 1968). Todos estos métodos de enseñanza comparten varios aspectos en común:a) realizan una retroalimentación inmediata y continua sobre el aprendizaje del material académico, b) las unidades de enseñanza se dividen en tantas partes como sea necesario para que el estudiante tenga un mejor manejo del material académico, c) el estudiante usa guías de estudio y tiene varias oportunidades de repetir quices y parciales, d) medición sistemáticamente delaprendizaje de cada una de las unidades de estudio, e) la estrategia de enseñanza se adapta a las condiciones particulares del estudiante y, f) la enseñanza de las unidades académicas está directamente ligada al contexto en el cual se da el entrenamiento (Bernstein \& Chase, 2013). Aunque estos métodos de enseñanza han mostrado eficacia en el ámbito académico (ver Moran \& Malott, 2004), presentan algunas dificultades, por ejemplo, el estudiante tiene que invertir mucho tiempo en la revisión del material académico para alcanzar los criterios de logro, al igual que el docente en el diseño de las clases. Además, ésta tecnología requiere que los docentes tengan un manejo básico en la aplicación de los principios operantes para maximizar sus efectos. Finalmente, por razones históricas y académicas, la tecnología operante produce reacción negativa en los docentes, lo que hace difícil su aplicación y masificación en el ámbito universitario (Moran \& Malott, 2004; Saville, Lambert, \& Robertson, 2011).

En las últimas décadas se ha venido investigando, dentro de la tradición operante, un método alternativo de enseñanza académica que supera varios de los problemas descritos anteriormente, y que se adapta al contexto educativo tradicional. Así mismo promueve la independencia y participación activa del estudiante en el proceso formativo (Brown, Killingsworth, \& Alavosius, 2014; Sturmey, Dalfen, \& Fienup, 2015). Este método de enseñanza universitaria se denomina interteaching (dada las dificultades de traducción se utiliza el nombre original en inglés) (Boyce \& Hineline, 2002). Los componentes esenciales son: a) guías de estudio, se entrega a los estudiantes una semana antes de clase una guía de estudio que contiene entre 8-12 preguntas de tipo conceptual y aplicado. Las preguntas están basadas en los objetivos del curso. Esta se trae a clase completamente terminada y sirve para facilitar la discusión por pares durante la clase, b) discusión por pares, durante la clase los estudiantes se reúnen en diadas para discutir sobre las diferentes preguntas de la guía. Esta se debe realizar durante dos terceras partes del tiempo de clase (e.g. $50 \mathrm{~min}$. en una clase de 75 min.). Igualmente el profesor se encarga de monitorear la discusión y responder a preguntas de los estudiantes, c) hoja de registro, en ésta los estudiantes describen cuales preguntas presentaron mayor dificultad y evalúan el tipo de discusión que tuvieron. El docente revisa ésta forma y desarrolla la siguiente clase basada en la dificultades descritas por los estudiantes, d) clase magistral, el docente presenta una clase corta donde clarifica los temas que no entendieron los estudiantes, esta debe durar aproximadamente una tercera parte de la clase total. Algunos investigadores han sugerido realizar la clase inmediatamente después de la discusión por pares (Boyce \& Hineline, 2002), mientras que otros proponen realizarla una semana después (Savilee $\&$ cols., 2011). Aspectos adicionales a la estrategia básica de interteaching incluyen puntos extras tanto por el tipo de discusión grupal realizada, así como por la participación en clase y entrega de guías (Brown \& cols., 2014; Saville \& cols., 2011; Sturmey \& cols., 2015).

Saville, Zinn, y Elliott (2005) realizaron el primer estudio sistemático de interteaching. En este los autores compararon tres estrategiaspara evaluar su efectividad sobre el desempeñoacadémico. En la primera condición los estudiantes fueron instruidos a leer un artículo, luego recibieron una clase magistral sobre el tema del mismo, y al final de la clasefueron evaluados. En la segunda condición los estudiantes recibieron una sesión de interteaching, como se describió anteriormente, y en la tercera condición, los estudiantes leyeron un artículo y luego fueron evaluados con un quiz sobre el mismo una semana después. Un grupo control fue también evaluado, este recibió únicamente un quiz. Los estudiantes en el grupo de interteaching presentaron puntajes más altos en los quices comparados con los otros grupos. Además no encontraron diferencias significativas en las notas de los quices entre los otros tres grupos. En un siguiente estudio, Saville, Zinn, Neef, Norman, y Ferreri. (2006) compararon de forma más sistemática la estrategia de interteaching con una de clase magistral tradicional. En este estudio alternaron ambas estrategias durante el semestre académico. Los resultados mostraron que tanto en la evaluación semanal, como final, los estudiantes pre- 
sentaron puntajes más altos en los quices tras las sesiones de interteaching. Estudios cuasi-experimentales (Emurian \& Zheng, 2010), experimentales (Filipiak, Rehfeldt, Heal, \& Baker, 2010), y con auto reportes (Sturmey \& cols., 2015) han evidenciado la efectividad del interteaching, comparado con métodos tradicionales de enseñanza, sobre la ejecución en evaluaciones semanales y semestrales en contextos universitarios.

A parte de investigar la efectividad del interteaching como estrategia para incrementar el rendimiento académico, también se han realizado estudios para evaluar la efectividad de sus diferentes componentes. Al respecto, varios investigadores han evaluado la calidad de los puntos asignados por participar dentro de los grupos (Rosales, Soldner, \& Crimando, 2014), efecto del tamaño del grupo sobre la discusión por pares (Truelove, Saville, \& Van Patten, 2013), efecto de la evaluación continua con quices sobre la ejecución en la evaluación final (Saville, Pope, Lovaas, \& Williams, 2012), y puntos extras por entregar las guías de estudio (Filipiak \& cols. 2010; Rehfeldt, Walker, Garcia, Lovett, \& Filipiak, 2010). A pesar que la investigación sobre la efectividad de los diferentes componentes del interteaching ha crecido en la última década (Brown \& cols., 2014; Sturmey \& cols., 2015), aún queda por investigar el efecto de otros componentes sobre el rendimiento académico.

Algunos investigadores han reportado que la discusión por pares sobre los temas de las guías al inicio de las clases es un componente esencial en el interteaching (Brown \& cols., 2014; Saville \& cols., 2011), sin embargo, a la fecha no se ha realizado algún estudio empírico que valide esta afirmación. Al respecto, Saville y cols. (2011) describen que los estudiantes generalmente reportan que no encuentran útil discutir por pares al inicio de la clase, debido a que las guías de estudio y clases clarifican todas las preguntas relacionadas al tema de ambas. Adicionalmente los estudiantes trabajan conjuntamente en el desarrollo de las guías, lo que disminuye potencialmente el efecto de la discusión por pares al inicio de la clase sobre el resultado de la evaluación semanal. De otra parte, aún existe desacuerdo entre los investigadores en interteaching sobre la relación entre las guías de estudio y la clase magistral donde se revisan los temas de la misma. Por ejemplo, Boyce y Hineline (2002) sugieren que los temas de la clase sean los mismos que los estudiantes discutieron en las guías esa sesión, sin embargo, Saville y cols. (2011) proponen que el docente se tome una semana preparando la clase y aclare las dificultades la siguiente sesión. Algunos estudios han realizado la revisión de los temas de las guías inmediatamente después de terminar la sesión de discusión por pares (Filipiak \& cols., 2010; Saville \& cols., 2005), mientras otros lo han hecho la siguiente semana (Rosales \& cols., 2014; Saville \& cols., 2006). En conclusión, aún se requiere más investigación para evaluar tanto el efecto de la discusión por pares, así como la relación entre las guías de estudio y la clase correspondiente al tema de las guías, sobre rendimiento académico en estudiantes universitarios. Por lo tanto, el objetivo de este estudio es evaluar el efecto de estos dos componentes dentro de la estrategia general de interteaching sobre las notas de las evaluaciones semanales.

\section{Método}

\section{Participantes}

Un total de 11 estudiantes universitarios de postgrado en psicología (10 mujeres y 1 hombre), con una edad promedio ( $M=26$ años) participaron en este estudio. La investigación se realizó con estudiantes que tomaron una clase de análisis conductual aplicado (ACA), ésta hacía parte del currículo académico de un programa de especialización en psicología clínica de la universidad donde se realizó el estudio. La clase tenía una duración semanal de una hora y media, y se desarrolló durante ocho semanas académicas. Un total de 14 estudiantes inscritos a la clase fueron invitados a participar voluntariamente en este estudio, durante la investigación tres abandonaron el curso, y solamente 11 lo terminaron. El contenido del curso se centró en aspectos teóricos y aplicados de ACA, así como en la selección y conceptualización de las diferentes técnicas comportamentales aplicadas a la psicología infantil. El estudio fue aprobado por el comité de ética de la universidad, y todos los participantes firmaron un consentimiento informadodonde se les indicaban las características del estudio.

\section{Diseño}

Se utilizó un diseño de tratamientos alternantes, el cual consistió en presentar de forma aleatoria y secuencial las condiciones de interteaching con y sin discusión por pares a cada uno de los participantes de la investigación durante un periodo de ocho semanas (Hayes, Barlow, \& Nelson-Gray, 1999). La condición de interteaching con discusión por pares (ICDPP) iniciaba con la entrega de una guía de estudio, los estudiantes la elaboraban y la traían completamente terminada a clase. Al inicio de la clase se reunían por parejas, discutían los temas de la guía, evaluaban la calidad de discusión de cada miembro de la pareja, y seleccionaban los temas de mayor dificultad a ser revisados por el docente. Inmediatamente después el docente revisaba estos temas en detalle en una clase magistral y terminaba la clase con un quiz evaluando los temas de la guía y clase. La condición de interteaching sin discusión por pares (ISDPP) fue idéntica a la anterior, con la única excepción que en esta los estudiantes no se reunían al inicio de la clase para discutir los temas de la guía. El docente iniciaba la clase y al final administraba el quiz a todos los estudiantes. Cada condición se presentó cuatro veces, de forma aleatoria, durante el transcurso de ocho semanas de clase. En la última semana se evaluó la efectividad de las estrategias y la metodología utilizada en ambas condiciones a través de un cuestionario de satisfacción administrado a todos los estudiantes. 


\section{Variables Dependientes}

Puntuación promedio en los quices. Esta variable se midió calculando el porcentaje promedio de las notas que obtuvieron los 11 participantes para cada uno de los cuatro quices asignados a cada condición durante el semestre. La escala de evaluación de los quices iba de 0 a 5 , estos posteriormente se transformaron a porcentajes. Todos los quices fueron similares tanto en la cantidad, como en el formato de las preguntas (respuesta corta, selección múltiple, falsa y verdadera, etc.). Los datos de los estudiantes que presentaron más de tres inasistencias se excluyeron del análisis final.

Notas Individuales en cada Condición. La escala de evaluación iba de 0 a 5 . Esta variable se midió calculando la nota promedio que obtuvo cada estudiante en los quices para cada condición experimental, es decir, se promediaron cuatro quices para cada participante por condición. Las notas de los quices de los estudiantes que se retiraron durante el transcurso de la investigación no fueron incluidas en esta medida.

Cuestionario de Satisfacción. En la última semana del semestre se les solicitó a los estudiantes responder a un cuestionario de validación social, que evaluóla utilidad, efectividad, y eficiencia de las diferentes estrategias utilizadas en cada una de las condiciones experimentales. Este cuestionario se desarrolló en una plataforma online especia- lizada en diseño de pruebas, el enlace fue enviado a cada uno de los estudiantes para que respondiera al cuestionario. Este cuestionario utilizó una escala Likert de 5 puntos donde se indica el nivel de satisfacción y utilidad del método de interteaching ( $0=$ "nunca", 1= "casi nunca", 2= "a veces", 3= "casi siempre", y 5= "siempre").

\section{Acuerdo Entre Observadores}

El segundo y tercer investigador del estudio de manera independiente calificaron el $100 \%$ de todos los quices y guías de estudio de las dos condiciones, ICDPP y ISDPP. EI acuerdo entre observadores (AEO) se calculó al evaluar el acuerdo en los puntos asignados a cada pregunta de los quices y guías de estudio. Este se obtuvo al dividir el número de acuerdos sobre el número de acuerdos más desacuerdos y el producto multiplicado por $100 \%$. Se consideró un acuerdo cuando ambos investigadores asignaban el mismo puntaje a las diferentes respuestas de los quices y guías de estudio. Por el contrario, se consideró un desacuerdo cuando los investigadores asignaban diferentes puntos a cada respuesta de los quices y guías de estudio. El acuerdo promedio fue de $94,7 \%$ para quices (rango, $86 \%$ a $100 \%$ ) y de $94 \%$ para las guías de estudio (rango, $90 \%$ a $100 \%$ )

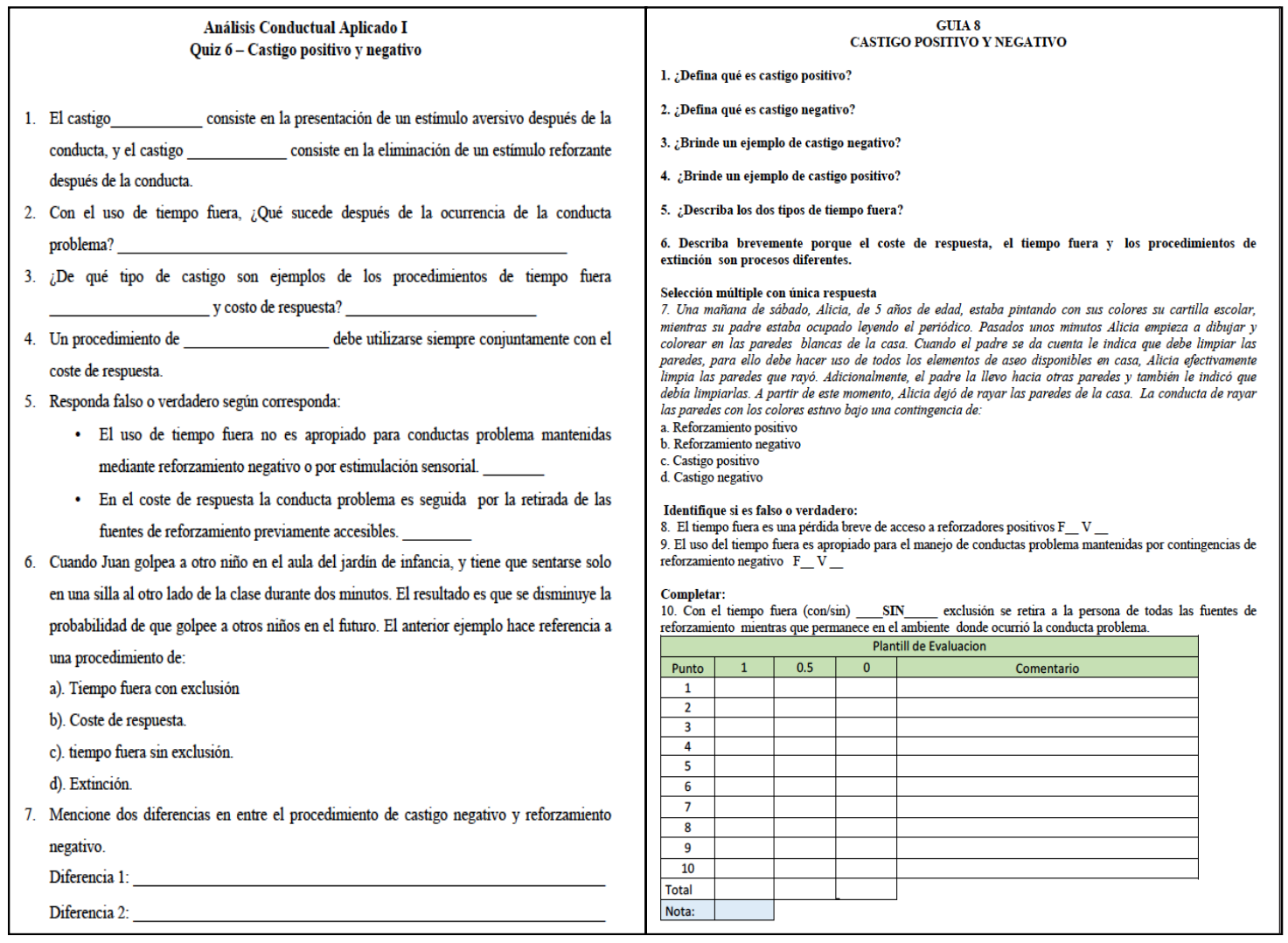

Figura 1. Ejemplo de una guía de estudio y su quiz respectivo. 


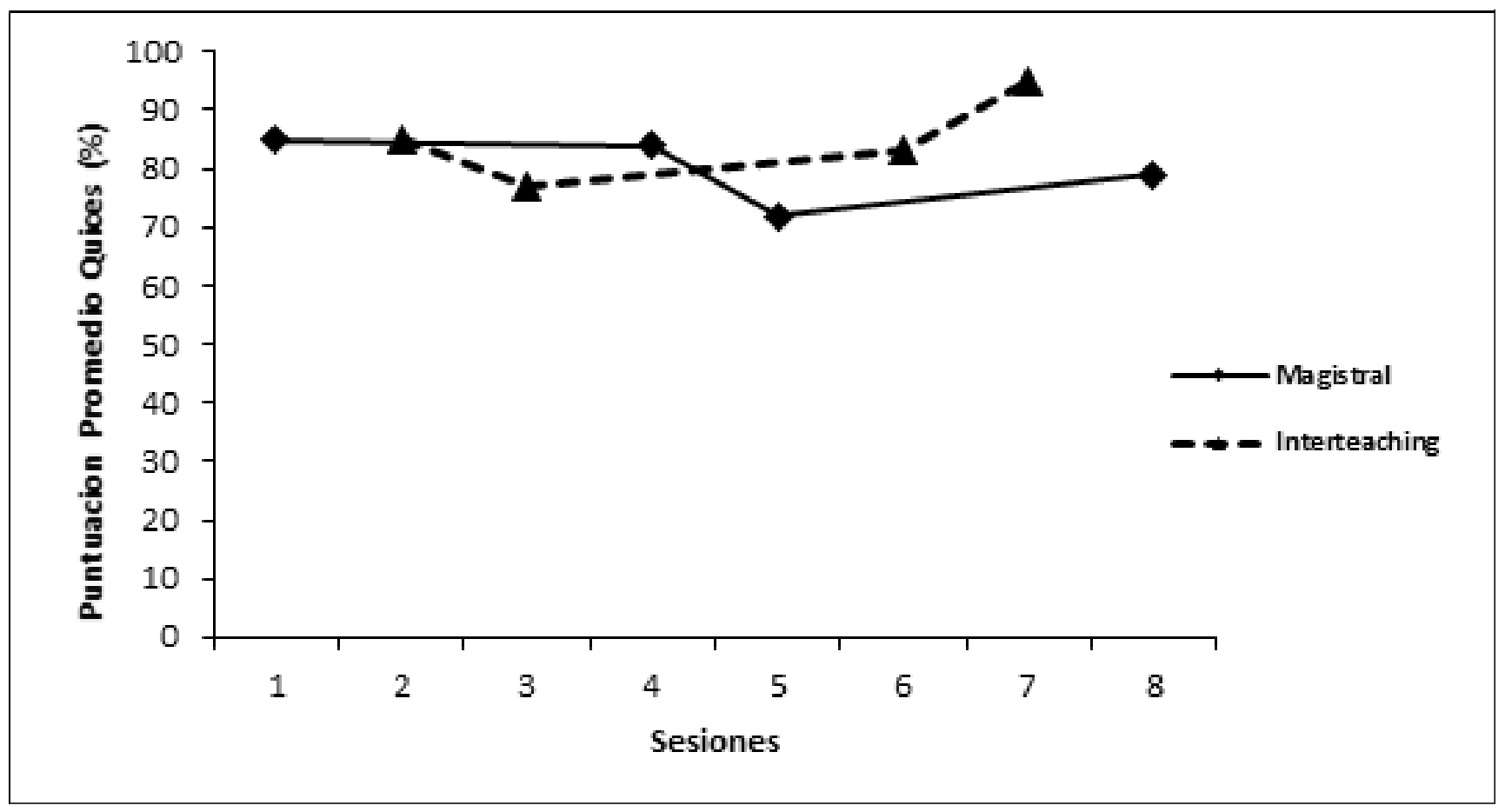

Figura 2. Esta gráfica muestra la puntuación promedio de quices media en porcentaje por cada una de las condiciones experimentales.

\section{Lugar y Materiales}

El estudio se llevó a cabo en un salón de clase, el cual disponía de las herramientas básicas para el proceso de enseñanza de los temas tratados (televisor, asientos, tablero, internet y computador). Los investigadores desarrollaron un total de ocho guías de estudio que contenían entre 8-12 preguntas de tipo conceptual y casos clínicos (ver Figura 1).

Las preguntas fueron elaboradas con base en las temáticas de la clase de ACA (e.g. definición, y aplicaciones de reforzamiento positivo y negativo, aspectos éticos de la aplicación de castigo positivo y negativo, función de la conducta, intervención funcional y control antecedente). El formato de presentación de la preguntas consistía en selección múltiple, falso y verdadero, completar y aplicaciones a casos particulares. Las guías estaban disponibles una semana antes de la clase en la plataforma virtual del curso, los estudiantes las podían descargar y responder a cada una de las preguntas antes de clase. Estas debían ser entregadas al primer investigador antes de iniciar la clase, luego la semana siguiente se regresaban a los estudiantes con la retroalimentación respectiva. Igualmente se elaboraron un total de 8 quices, que contenían entre 6-7 preguntas, estos presentaban variaciones mínimas a las preguntas de las guías (ver Figura 2). Estos se administraban los último diez minutos de la clase, y al igual que las guías, los estudiantes recibían retroalimentación semanal por la ejecución en los mismos.

\section{Procedimiento}

Los estudiantes fueron informados al inicio del semestre sobre las dos condiciones experimentales que se iban a alternar durante el semestre, sin embargo el propósito del estudio no fue descrito en detalle para evitar cualquier sesgo experimental. Cada una de las dos condiciones fueron aleatorizadas previamente antes de iniciar el estudio. Un total de cuatro sesiones por condición fueron distribuidas a través del semestre, y a cada una se le asignó una guía de estudio y un quiz de evaluación.

Interteaching con discusión por pares. Esta condición iniciaba con la entrega de una guía de estudio a cada uno de los estudiantes, ellos contaban con una semana para entregarla completamente terminada al docente antes de iniciar la siguiente clase. Un total de cuatro temas fueron asignados aleatoriamente a esta condición (conducta blan$\mathrm{co}$, reforzamiento positivo y negativo, y análisis funcional), cada uno con sus respectivas guías y quices. Los estudiantes debían de traer una copia de la guía y reunirse en parejas para discutir durante los primeros 15 minutos de clase los diferentes temas evaluados en la guía. Estas parejas se rotaban semanalmente para prevenir cualquier habituación o sesgo experimental. Durante las sesiones de discusión el docente hacía entrega a los estudiantes de una formato de evaluación, en este ellos debían de evaluar los temas que presentaban mayor dificultad, temas particulares de la guía que les gustaría ver en clase, preguntas que le quitarían a la guía, nivel de preparación y discusión que tenía el compañero durante este espacio y comentarios generales. 
Durante estos 15 minutos los estudiantes recibían apoyo y retroalimentación sobre los temas por parte del docente y los otros dos coinvestigadores. Durante la sesión de discusión, si los estudiantes hablaban de otros temas diferentes al de las guías, el profesor o asistente los redirigían al tema evaluado en la guía. Una vez terminado el tiempo, uno de los coinvestigadores procedía a escribir en el tablero los temas que mayor dificultad presentaban los estudiantes en las guías. Estos temas se organizaban en secuencia y el docente procedía a explicar cada uno de ellos durante la siguiente hora de clase. A diferencia del procedimiento tradicional de interteaching, en este estudio los temas presentados en clase correspondían a la guía evaluada para esa sesión. En decir, los estudiantes no esperaban una semana pare recibir la clase correspondiente al tema de la guía. Una vez concluido el tema, se procedía a presentar un quiz los últimos diez minutos. Los quices contenían preguntas relacionadas con las guías de estudio. La sesión de interteaching terminaba con la entrega del quiz al docente.

Interteaching sin discusión por pares. Esta condición iniciaba de la misma forma que la anterior, es decir, con la entrega de la guía de estudio a los estudiantes, y el regreso de esta completamente terminada al docente antes de iniciar la siguiente clase. A esta condición se le asignaron aleatoriamente cuatro temas diferentes (registros de conducta, acuerdo entre observadores, control antecedente y análisis funcional descriptivo), y fueron igualmente distribuidos aleatoriamente a través de las ocho sesiones experimentales (ver figura 1). La duración de la clase era de 1 hora y 20 minutos, y los últimos 10 minutos fueron asignados para responder a las preguntas del quiz. A diferencia de la condición anterior, esta iniciaba directamente con la clase magistral del docente, quien revisaba los diferentes temas asignados para esa semana. El docente iniciaba la clase con la revisión de conceptos claves de cada uno de los temas, luego hacía preguntas a los estudiantes sobre estos, y finalmente realizaba ejemplos con cada uno de los temas. Durante este tiempo los estudiantes tenían la oportunidad de hacer preguntas sobre los temas y revisar videos y aplicaciones de los conceptos a casos clínicos reales. Esta sesión terminaba con la administración de un quiz a cada uno de los estudiantes sobre los temas de la guía y los revisados en clase.

\section{Resultados}

\section{Porcentaje promedio enquices}

En la figura 2 se puede observar el porcentaje promedio de preguntas resueltas correctamente para la condición de ICDPP (sesiones 1, 4, 5 y 8), e ISCPP (sesiones 2, 3, 6 y 7). Durante la condición de ISDPP los estudiantes obtuvieron un porcentaje promedio más bajo en las notas de los quices $(M=79,5 \%, D E=7,11)$ comparado con las notas en los quices durante la condición de ICDPP $(M=84 \%, D E=$ $5,74)$. Para evaluar las diferencias estadísticas entre ambas condiciones se procedió a realizar prueba t para muestras pareadas, los resultados muestran que las notas en los quices de la condición de ICDPP fueron significativamente más altas que en la otra condición $t(10)=-2.135, p<0.05$.

\section{Notas Promedio de Cada Sesión en Ambas Condiciones}

La escala de evaluación utilizada para los quices iba de 0 a 5 . El promedio de notas para las cuatro sesiones de ISDPP arrojó los siguientes resultados, primera sesión $(M=$ 4.2; $D E=0.76)$, cuarta $(M=4.2 ; D E=0.74)$, quinta $(M=3.6$; $D E=0.66)$, y octava $(M=3.9 ; D E=0.92)$. En la condición de ICDPP el promedio de notas en la segunda sesión $(M=4.2$; $D E=0.58)$, tercera $(M=3.9 ; D E=0.86)$, sexta $(M=4.0 ; D E$ $=0.86)$, y séptima $(M=4.7 ; D E=0.5)$. El promedio de notas en la condición de ICDPP fue más alta $(M=4.2 ; D E=0.51)$, comparada con la condición de ISDPP $(M=3.9 ; D E=0.4)$.

\section{Cuestionario de Satisfacción}

La escala del cuestionario de satisfacción iba de 0 a 4, donde (0= "nunca", 1= "casi nunca", 2= "a veces", 3= "casi siempre", y $5=$ "siempre"). Se presenta la mediana obtenida en cada una de las preguntas y los rangos de los puntajes. A la primera pregunta "disfrute más las sesiones de clase donde no se realizó la revisión de temáticas en parejas" los estudiantes respondieron con una mediana $(M=1$, rango $0-4)$, y en la segunda pregunta "encontré más útil discutir las guías de estudio con un compañero de clase" ( $M=2$, rango 2-4). Respecto a la tercera pregunta, "disfrute más las clases que tenían guías y selección de temas de clase" la mediana ( $M=4$, rango 3-4) fue alta, al igual que en la cuarta pregunta "las guías fueron útiles para obtener notas altas en los quices" ( $M=4$, rango 2-4). En la quinta pregunta "prefieres estudiar por tu cuenta sin el apoyo de guías"solo un estudiante respondió casi siempre $(M=0$, rango $0-3)$, mientras en la sexta pregunta "pude seguir adecuadamente la estructura de la clase" la mediana fue alta $(M=3$, rango $2-4)$. La mediana en la séptima pregunta "las guías de estudio contribuyeron en tener más elementos para la discusión en clase", la mayoría de estudiantes reportaron total satisfacción $(M=4$, rango 3-4). En la octava pregunta "me gustó formar grupos de discusión al iniciar la clase" y novena "el tiempo invertido en completar las guías fue suficiente" obtuvieron una mediana $(M=3$, rango $2-4)$ y $(M=3$, rango $2-4)$, respectivamente. Finalmente a la última pregunta "utilizaste las guías como material de estudio para los quices y parciales" la mediana $(M=4$, rango 3-4) fue alta.

\section{Discusión}

Los resultados de este estudio muestran que el procedimiento de interteaching con discusión por pares (ICDPP) 
fue significativamente más efectivo que la condición de interteaching sin discusión por pares (ISDPP) en el incremento del rendimiento académico evaluado a través de quices semanales durante ocho semanas. Específicamente, los resultados muestran que la discusión por pares es un componente esencial en el éxito y eficacia de las sesiones de interteaching. Al igual que evidencianlos modelos constructivistas de la educación, esta investigación muestra que la participación activa de los estudiantes es un factor determinante en la construcción y apropiación del conocimiento (Burkholder \& Peláez, 2000). Igualmente los resultados muestran, medido a través de la evaluación directa y cuestionario de satisfacción, que los estudiantes encontraron más efectivo revisar los temas de la guía inmediatamente después en la misma clase, que esperar una semana después. Estos datos concuerdan con estudios anteriores (Filipiak \& cols., 2010; Saville \& cols., 2005) respecto a la preferencia y efectividad de revisar los temas de las guías la misma sesión. En conclusión los resultados de este estudio validan la importancia de la discusión por pares dentro del método en interteaching y la necesidad de revisar los temas de la guía la misma sesión de clase (Brown \& cols., 2014; Saville \& cols., 2011).

Es importante mencionar que a pesar que los resultados de los quices dentro de la condición de ICDPP fueron significativamente más altos que en la otra condición, no se puede concluir taxativamente que esta es una condición sine qua non dentro del interteaching. La literatura experimental avala el rol de la discusión activa entre estudiantes en el rendimiento académico (Ruiz-Primo \& cols., 2011), sin embargo, los resultados de este estudios deben tomarse con precaución ya que varios aspectos no estuvieron bajo control experimental. Por ejemplo, no se controló el tipo y calidad de discusión que los estudiantes realizaron por parejas, algunos estudiantes pudieron dedicarse a hablar de otros temas durante esta sesión. Igualmente el trabajo en equipo en el desarrollo de las guías pudo servir como un elemento preparatorio para los quices, dejando la discusión por pares solo en un elemento de trámite. Finalmente, algunos estudiantes tenían mayor conocimiento del tema de clase, lo que pudo influir en la media general del rendimiento académico. Los resultados de este estudio suman a la evidencia general de la efectividad de otros componentes del interteaching sobre el rendimiento académico (Rehfeldt \& cols., 2010; Rosales \& cols., 2014; Saville \& cols., 2012; Truelove \& cols., 2013), sin embargo es importante replicar estos estudios con una muestra más grande y ejercer mayor control experimental.

Con respecto a los datos del cuestionario de satisfacción, estos muestran varios aspectos importantes a mencionar. A las preguntas sobre la incidencia de las guía en la participación en clase, notas en los quices, herramienta de estudio y selección de los temas de clase, la mediana general fue cuatro, es decir, los estudiantes en su mayoría mostraron satisfacción por la utilidad y efecto de estas sobre el rendimiento académico, y comprensión del tema. Estos datos muestran que las guías sirven como un elemento motivacional en la participación activa en clase, así como una herramienta para enfrentar con menor estréslos temas difíciles en clase (Vandsburger \& Duncan-Daston, 2011). Los estudiantes evaluaron con una mediana de tres, aspectos como la reunión por pares antes de la clase y el tiempo invertido en completar las guías. Es decir, una parte del grupo de estudiantes encontró poco útil reunirse en grupos para descurtir los temas de la guía, así como el tiempo que tuvieron que invertir en el desarrollo de la misma. Algunos estudiantes reportaban que realizar las guías les quitaba tiempo para desarrollar otras actividades académicas, sin embargo las encontraban útiles. Un aspecto a revisar en investigaciones futuras es el número de preguntas en las guías. Igualmente, como se mencionó en el párrafo anterior, es importante aplicar contingencias particulares a la discusión en grupo para que los estudiantes no encuentren este componente del interteaching aversivo (ver Rosales \& cols., 2014; Saville \& cols., 2011 para datos sobre estos aspectos). En general, los estudiantes reportaron que las guías fueron elementos útiles en la comprensión del tema y sirvieron para incrementar su rendimiento académico, sin embargo, encontraron que la reunión por pares y tiempo invertido en el desarrollo de las guías fue poco útil.

Una limitante de este estudio tuvo que ver con que algunas clases en la condición de ICDPP no se pudieron terminar completamente. En algunas sesiones los estudiantes sugerían varios temas que superaban el tiempo disponible para revisarlos a profundidad, esto llevó a que temas particulares no se revisaran completamente. Esta limitación se sumaba al hecho que algunos estudiantes no tenían la formación académica mínima en los principios y procedimientos de ACA y retrasaban el avance a otros temas. Una limitante importante en este estudio fue el número de sesiones asignadas a cada condición. El total de semanas asignadas al curso de ACA era de catorce, sin embargo solo se llevaron a cabo ocho, debido a que algunos estudiantes se inscribieron a la clase en la segunda y tercera semana del semestre. Igualmente se eliminó un tema de los datos finales, debido a que en esta no se pudo administrar el quiz. Las semanas totales también se vieron recortadas por que los temas evaluados al inicio del semestre eran de tipo filosófico y conceptual. Solo se incluyeron dentro de las ocho semanas temas directamente relacionados con los principios y procedimientos de ACA. Finalmente la generalidad de los resultados se ve minimizada debido a lo pequeña de la muestra.

Debido a las limitaciones de este estudio se sugieren algunas alternativas para mejorar su control experimental. Por ejemplo, es importante evaluar si otros componentes como la calidad de los puntos asignados a la discusión por pares (Rosales \& cols., 2014), guías (Filipiak \& cols., 2010), tamaño total del grupo (Saville \& cols., 2011), y evaluación semanal (Saville \& cols., 2012), tienen el mismo efecto sobre las notas en los quices cuando estos se combinan con y sin discusión por pares. Igualmente es relevante revisar los mismos componentes cuando los temas de la guía se revisan inmediatamente después en la clase. También es importante evaluar estos dos componentes, discusión por pares y clase, sobre el desarrollo de pensamiento crítico (Saville, Zinn, Lawrence, Barron, \& Andre, 2008) y rendimiento académico en 
diversos currículos (Schneider \& Goto, 2009; Slagter \& Scribner, 2014; Tsui, 2010). Finalmente es importante adaptar el interteaching a plataformas virtuales para evaluar si tiene el mismo efecto que el interteaching tradicional (Emurian \& Zheng, 2010).

En conclusión, este estudio muestra que la discusión por pares e inmediatez de la discusión de los temas de la guía por parte del docente, son un elemento central en la efectividad del interteaching y rendimiento académico. Aunque este estudio presenta algunas limitaciones, es importante mencionar que en general los datos son congruentes con los que muestra la investigación general en interteaching (Brown \& cols., 2014; Filipiak \& cols., 2010; Rehfeldt \& cols., 2010; Rosales \& cols., 2014; Saville \& cols., 2011; Sturmey \& cols., 2015). Se requiere más investigación en el futuro para evaluar la generalidad de esta metodología a diferentes campos de la educación, así como la evaluación de sus diferentes componentes. El interteaching muestra que el estudiante es un elemento activo en el proceso formativo y las estrategias operantes tradicionales como reforzamiento o modelamiento están al servicio de este proceso.

\section{Referencias}

Bernstein, D. J. \& Chase, P. N. (2013). Contributions of behavior analysis to higher education. Em G. Madden (Org.), APA handbook of behavior analysis: Volume 2 Translating principles into practice (pp. 523-543). Washington, D.C.: APA Press.

Boyce, T. E. \& Hineline, P. N. (2002). Interteaching: A strategy for enhancing the user-friendliness of behavior a arrangements in the college classroom. The Behavior Analyst, 25(2), 215-226.

Brown, T. W., Killingsworth, K., \& Alavosius, M. P. (2014). Interteaching: An evidence-based approach to instruction. International Journal of Teaching and Learning in Higher Education, 26(1), 132-139.

Burkholder, E. O. \& Peláez, M. (2000).A behavioral interpretation of Vygotsky's theory of thought, language, and culture. Behavioral Development Bulletin, 9(1), 7.

Emurian, H. H. \& Zheng, P. (2010). Programmed instruction and interteaching applications to teaching $\mathrm{Java}^{\mathrm{TM}}$ : A systematic replication. Computers in Human Behavior, 26(5), 1166-1175.

Ferster, B. (2014). Teaching machines: Learning from the intersection of education and technology. Baltimore, MD: Johns Hopkins University Press

Filipiak, S. N., Rehfeldt, R. A., Heal, N. A., \& Baker, J. C. (2010). The effects of points for preparation guides in interteaching procedures. European Journal of Behavior Analysis, 11,115-132.

Hayes, S. C., Barlow, D. H., \& Nelson-Gray, R. O. (1999). The scientist practitioner: Research and accountability in the age of managed care(2nd ed.). Needham Heights, MA: Allyn \& Bacon.

Henklain, M. H. O. \& Carmo, J. S. (2013). Contribuições da análise do comportamento à educação: Um convite ao diálogo. Cadernos de Pesquisa, 43(149), 704-723.

Kara, N. \& Sevim, N. (2013). Adaptive learning systems: Beyond teaching machines. Contemporary Educational Technology, 4(2), 108-120.

Keller, F. S. (1968). Goodbye, teacher. Journal of Applied Behavior Analysis, 1, 79-89.

Lindsley, O. R. (1991). Precision teaching's unique legacy from BF Skinner. Journal of Behavioral Education, 1, 253-266.

Moran, D. J. \& Malott, R. W. (Orgs.). (2004). Evidence-based educational methods. San Diego: Elsevier Academic Press.

Rehfeldt, R. A., Walker, B., Garcia, Y., Lovett, S., \& Filipiak, S. (2010). A point contingency for homework submission in the graduate school classroom. Journal of Applied Behavior Analysis, 43(3), 499-502.

Rehfeldt, R. A., Jung, H. L., Aguirre, A., Nichols, J. L., \& Root, W. B. (2016). Beginning the dialogue on the e-transformation: Behavior analysis' first massive open online course (MOOC). Behavior Analysis in Practice, 9, 3-13.

Rosales, R., Soldner, J. L., \& Crimando, W. (2014). Enhancing the impact of quality points in interteaching. Journal of the Scholar ship of Teaching and Learning, 14(5), 1-11.

Ruiz-Primo, M. A., Briggs, D., Iverson, H., Talbot, R., \& Shepard, L. A. (2011). Impact of undergraduate science course innovations on learning. Science, 331 (6022), 1269-1270.

Saville, B. K., Lambert, T., \& Robertson, S. (2011). Interteaching: Bringing behavioral education in to the 21st century. The Psychological Record, 61(1), 153-166.

Saville, B. K., Pope, D., Lovaas, P., \& Williams, J. (2012). Interteaching and the testing effect: A systematic replication. Teaching of Psychology, 39(4), 280-283.

Saville, B. K., Zinn, T. E., \& Elliott, M. P. (2005). Interteaching versus traditional methods of instruction: A preliminary analysis. Teaching of Psychology, 32,161-163.

Saville, B. K., Zinn, T. E., Lawrence, N. K., Barron, K. E., \& Andre, J. (2008). Teaching critical thinking in statistics and research methods. Em D. S. Dunn, J. S. Halonen, \& R. A. Smith (Orgs.), Teaching critical thinking in psychology: A handbook of best practices (pp. 149-160). Malden, MA: Wiley-Blackwell.

Saville, B. K., Zinn, T. E., Neef, N. A., Norman, R. V., \& Ferreri, S. J. (2006). A comparison of interteaching and lecture in the college classroom. Journal of Applied Behavior Analysis, 39(1), 49-61. 
Schneider, J., \& Goto, K. (2009). Interteaching: A new innovative approach to facilitate university student learning in the field of nutrition. Journal of Nutrition Education and Behavior, 41(4), S10.

Slagter, T. H., \& Scribner, D. L. (2014). Interteach and student engagement in political science. Journal of Political Science Education, 10(1), 81-92.

Sturmey, P., Dalfen, S., \& Fienup, D. M. (2015). Inter-teaching: A systematic review. European Journal of Behavior Analysis, 16(1), 121-130.
Truelove, J. C., Saville, B. K., \& Van Patten, R. (2013). Interteaching: Discussion group size and course performance. Journal of the Scholarship of Teaching and Learning, 13(2), 23-30.

Tsui, M. (2010). Interteaching students as teachers in lower-division sociology courses. Teaching Sociology, 38(1), 28-34.

Vandsburger, E. \& Duncan-Daston, R. (2011). Evaluating the study guide as a tool for increasing students' accountability for reading the textbook. Journal of College Reading and Learning, 42(1), 6-23.

Recebido em: 14/07/2015

Reformulado em: 30/05/2016

Aprovado em: 24/06/2016

\section{Sobre os autores}

Yors A. Garcia (yorsgarcia@yahoo.com)

Ph.D. BCBA-D, Fundacion Universitaria Konrad Lorenz

Laurent Orozco (laurentorozco@hotmail.com)

Fundacion Universitaria Konrad Lorenz

Giouvanni Martin (giomar66@gmail.com)

Fundacion Universitaria Konrad Lorenz 